\title{
Le ventre dans l'écriture de Sony Labou Tansi et Tchicaya U Tam'si
}

Notes pour une anthropologie génétique

Patrice Yengo

\section{(2) OpenEdition}

Journals

Édition électronique

URL : https://journals.openedition.org/coma/238

DOI : $10.4000 /$ coma.238

ISSN : 2275-1742

Éditeur

Institut des textes \& manuscrits modernes (ITEM)

Référence électronique

Patrice Yengo, «Le ventre dans l'écriture de Sony Labou Tansi et Tchicaya U Tam'si », Continents manuscrits [En ligne], 1 | 2014, mis en ligne le 22 avril 2014, consulté le 12 janvier 2023. URL : http:// journals.openedition.org/coma/238; DOI : https://doi.org/10.4000/coma.238

Ce document a été généré automatiquement le 12 janvier 2023.

\section{(c) $($ () $\ominus$}

Creative Commons - Attribution - Pas d'Utilisation Commerciale - Pas de Modification 4.0 International - CC BY-NC-ND 4.0

https://creativecommons.org/licenses/by-nc-nd/4.0/ 


\section{Le ventre dans l'écriture de Sony Labou Tansi et Tchicaya U Tam'si}

Notes pour une anthropologie génétique

\section{Patrice Yengo}

1 Dans quelle langue s'exprime un écrivain africain francophone? Telle est la question qui surgit, récurrente, lorsqu'au détour d'une phrase dans un texte, l'on est confronté à un mot, une tournure de phrase, une forme de style que l'on croit pouvoir attribuer à la langue maternelle de l'auteur. On s'imagine alors que le premier exercice auquel s'est livré ce dernier est une simple traduction. Alors qu'on est loin du compte. Car la pluralité de sens est originelle et constitutive de la richesse du texte, et le transport de sens d'une langue à l'autre produira du sens nouveau à l'intersection de plusieurs niveaux de signification. Ainsi, à l'évidence, l'auteur francophone n'est pas un simple traducteur; son positionnement d'écrivain dans une langue qui n'est pas la sienne brouille les contours de cette fonction.

2 On voudrait donc ici rendre compte de cette dynamique singulière, à partir du champ sémantique du ventre, central dans l'œuvre de Tchicaya U Tam'si et de Sony Labou Tansi, auquel ces deux auteurs ont consacré deux de leurs ouvrages au titre homonyme, le Ventre et Sa majesté le ventre ${ }^{1}$, dont l'avant-texte est tout simplement intitulé Le Ventre avant que Sony n'ajoute à la main Sa majesté.

\section{Origine du ventre, ventre des origines}

3 En réponse à Edouard Maunick qui l'interrogeait sur les ressorts de son écriture, Sony Labou Tansi eut tôt fait de placer le lieu de son inspiration dans le ventre :

D'une manière ou d'une autre, il m'est possible de répondre à toutes les questions qu'on pourrait me poser sur mon verbe en une seule et unique phrase: je suis écrivain, que voulez-vous que j'y fasse, les choses viennent du ventre. Parce que le ventre demande un peu de place sur notre terre en question. Parce que les gens semblent ignorer que c'est de la viande que nous vient la vie ${ }^{2}$. 
Le lecteur averti de La Vie et demie, son premier roman dans lequel il avait déjà mis "son ventre, ses entrailles, sa chair ${ }^{3}$ ", croit trouver dans cette réplique une confirmation : par la façon dont celui-ci torture les mots, les dissocie ou leur fait rendre gorge, et surtout par la manière toute viscérale qu'a son écriture de montrer l'atelier du ventre à l'œuvre, avec ses déjections et ses humeurs viciées qui émaillent ses romans, Sony est celui qui fait «prendre ventre ${ }^{4}$ » au mot afin de mieux l'incruster dans la chair.

Le ventre est le lieu de cette élaboration, l'horizon avec lequel il faut composer, imposer la vie et ses mots et surtout ce qui gît sous les vies et les mots : «La vie. C'est toujours lourd. Les mots aussi. Mais maintenant, ce qui compte [...] c'est ce qui dort sous les mots [...]; ce qu'il y a sous les vies ", nous dit Dadou dans L'Anté-Peuple. Il se trouve que sous les mots gisent des indications subliminales qu'il appartient au poète seul de décoder. Sony nous avertit : son écriture « est une manière de se tenir le ventre avant la tête ${ }^{5}$ ». Du moins y fait-il directement allusion dans l'interview qu'il accorda à équateur, une revue éphémère qui lui consacra son premier numéro :

Je stipule que le véritable débat de notre siècle n'est pas dans le crâne.

Il est où?

Au ventre, au sens que nous autres Africains donnons à ce mot $^{6}$.

Quel est donc ce sens que les Africains donnent à ce mot? Sony Labou Tansi esquisse une première réponse: «Les Européens pensent qu'ils gardent l'Homme dans la tête, les Africains qu'ils l'ont dans le cul. Je pense quant à moi que l'Homme est dans le coeur, nous disons ventre ${ }^{7} »$.

6 Est-ce à dire que les deux termes, cœur et ventre, sont équivalents? L'approche anthropologique est celle qui nous parait la plus appropriée pour traquer la pluralité des acceptions que ce mot recèle dans les langues bantoues du Bassin du Congo. Elle peut nous aider à comprendre quel sens particulier, par exemple, voyage à l'intérieur d'un segment textuel. Lequel sens peut lui-même prendre une autre direction dans un autre fragment ou jouer de duplicité. Cela permet de baliser le chemin de la critique génétique proprement dite, dans le domaine africain, notamment, où les manuscrits sont voués à une disparition quasi programmée. C'est une approche qui peut, en outre, s'enrichir des rapports avec la sociocritique ou la sémiotique par exemple.

\section{Moyo, Vumu : les deux ventres}

Dans le bassin du Congo, deux termes cohabitent en kikongo pour désigner le ventre: Moyo et Vumu. Souvent confondus, ces deux vocables ont été réduits à l'usage au seul Moyo. Or ce terme est polysémique puisqu'il définit d'un côté le clan et la force vitale qu'elle abrite, et que de l'autre, il sert à nommer l'abdomen et le cœur, comme le rappelle Janzen: "Moyo (life force as synonyme for both vumu (abdomen) and ntima $\left(\right.$ heart $\left.{ }^{8}\right) »$. En fait, la polysémie n'est qu'apparente. Vumu est un terme anatomophysiologique alors que Moyo est le ventre symbolique, celui qui donne naissance non seulement au sujet individuel mais aussi à sa lignée. Et comme tel, il est le lieu qui abrite la force vitale protectrice du groupe en même temps qu'il est l'âme et le cœur du clan. La force vitale est la somme des énergies circulant à l'intérieur du ventre-clan figuré par son représentant qu'est l'oncle maternel. Dans ces sociétés essentiellement matrilinéaires, l'oncle est de la cognation des ancêtres et, dans l'ordre du lignage, leur émanation, dont il assure le désir d'ordre et l'ordre des désirs du clan.

Continents manuscrits, 1 | 2014 
Moyo, une longue chaîne symbolique s'organise autour de ce qui compose le ventre. En partant de la perspective lignagère, celle-ci procèderait de sa source clanique, réalité première du ventre, qui irriguerait immédiatement une autre réalité d'ascendance nouvelle, que l'on ne saurait nommer autrement que «ventre national».

S'emboîtent ainsi diverses amplitudes du ventre, qu'on prend le parti de distinguer par ventre d'avant le Ventre, pour désigner la réalité clanique, et par Ventre pour rendre compte de la réalité nationale. Le monde de l'attachement aux racines cède la place à la conception d'une réalité qui advient. Celle-ci ne relève pas de la substantification d'un mythe fondateur mais d'un évènement : le ventre procède dorénavant d'un évènement historique, la colonisation. Ce qui était du clan se métamorphose au cours de la mutation des indépendances dans le devenir national de ce ventre organique, dont l'enjeu est d'inscrire les ventres-clans parcellisés dans une histoire unique, celle d'un nouveau Moyo, à la tête duquel la place avunculaire est dorénavant occupée par la figure fondatrice du héros de l'Indépendance. Lequel, nouveau porteur de ventre, ne tire pas sa légitimité de sa position au niveau du clan, ventre d'avant le Ventre, mais de l'acte de fonder, qui s'est détaché de lui pour l'instituer autant en héros qu'en ancêtre ${ }^{9}$ du lignage d'État.

La notion de ventre qui, dans le clan, était indissociable de «l'image de la mort, dans laquelle se fixent les symboles clés de l'organisation sociale à travers les oppositions fondamentales mais structurantes entre le monde des vivants et le monde des morts ${ }^{10} "$, mais aussi de l'imaginaire de la sorcellerie, se maintient alors dans la figure du héros, au prix d'une mort programmée.

11 C'est également l'exercice auquel se livre Tchicaya U Tam'si dans Le Ventre, qui met en relation la figure fondatrice tragique de Lumumba et le motif récurrent du Ventre.

L'écriture de ce recueil poétique nous montre à quel point les images du ventre recomposent aussi bien les réalités de l'anatomie et de la physiologie - «C'est moi qui tire / sur les viscères / de ce ventre qui tremble pour mon pardon ${ }^{11}$ ", y déclare l'auteur -, que l'engagement dans le ventre fantasmatique de l'unité nationale - «Le mot d'ordre est de vivre / le cœur à gauche ${ }^{12}$ ! », y note-t-il encore.

13 Sony Labou Tansi parle, lui, de "panse à gauche ${ }^{13}$ » et nous éclaire, par son écriture humorale, sur la portée de ce projet corporel dont les postures scatologiques émaillent ses romans, de La Vie et demie au posthume Le Commencement des douleurs, en passant par L'État honteux dont la version originale, Machin la Hernie ${ }^{14}$, rétablit le corps « hernié » du pouvoir dans ses attributions de ventre terminal de l'analité et de la castration.

14 Tchicaya U Tam'si s'interroge: "comment veux-tu voir mon ventre? / Au profil, il avait le bec/de mon hernie ombilicale ${ }^{15} »$. La réponse indique clairement qu'il se prononce d'emblée pour une anatomie de la douleur et de l'engagement, qu'il particularise dans la mise en relief de la vie interne du ventre en donnant à sa douleur une charge libidinale: «Ah! Je puis être votre très humble serviteur parce que par cette crevasse dans votre ventre s'échappe le chant de mes tripes ${ }^{16} . . . »$.

Il s'ensuit que le ventre peut se recentrer sur des gnosies corporelles, à partir d'une symptomatologie propre, presque sorcière - «Je mange les entrailles / de mon pays maudit ${ }^{17}$ ", déclare l'auteur -, où ce qui est appréhendé à partir du schéma corporel du ventre individuel ${ }^{18}$ concerne d'abord la présence du ventre social éprouvé et pour lequel comptent aussi les afférences douloureuses des expériences passées de 
l'histoire : d'une histoire congestionnée par de lâchetés domestiques que la Cléopâtre de SLT stigmatise ouvertement : "Ouvrez mes intestins / donnez-les à manger à tous les chiens de la terre [...] Quoi / Vous voudriez que pour / vous dérober à votre couardise / je me change prophète / femelle ${ }^{19}$ ? [...]» : une réplique à laquelle semble répondre en écho cette strophe du Ventre de Tchicaya : «Mes viscères se syndiquent / à l'ordre que l'on y trouvera / la débâcle est à craindre ${ }^{20}$ ».

Un premier constat s'impose : même si le règne du signifiant-maître de la colonisation est derrière nous, l'écriture de Tchicaya U Tam'si et Sony Labou Tansi s'invente dans la prégnance du ventre et de la puissance du Moyo face au surmoi colonial organisé autour de l'interdit, de l'œdipe et du meurtre. Aussi décomposent-ils le corps du langage comme ils le font pour le ventre, afin de mieux déchiffrer, dans l'ordonnancement premier de ses organes (foie, poumons, cœur, estomac...), la non-contradiction entre l'indivision du ventre et la multiplicité des organes qui l'emplissent et en font le premier empire de l'âme ${ }^{21}$.

17 Cela permet, en jouant des synonymies, des répétitions, voire des redondances, d'user non seulement de redoublements de sens dans le passage d'un organe ou d'une fonction à l'autre - "Tout mon cœur vous a porté au ventre ${ }^{22}$ », déclare ainsi TUT-, mais également de :

- retisser les réseaux des sens oblitérés - «Le foie pour quel excès de vin ? La foi en quel corps en holocauste ? M'en laissera-t-on voir le ventre ${ }^{23}$ ? ", s'interroge l'auteur - ;

- ou, chez Sony Labou Tansi, de renouer avec les relations refoulées de son corps lexical: «Pour réfléchir je me prends les boyaux, le ventre si vous voulez. Et parfois je vais plus loin je me prends le sexe, la braguette si vous voulez », note-t-il dans son agenda ${ }^{24}$.

\section{Dynamique du ventre}

On connait la boutade de Tchicaya U Tam'si répondant à l'un de ses compatriotes qui lui reprochait son long exil : «Vous habitez le Congo, le Congo m'habite ${ }^{25}$ ».

Le ventre comme métaphore du pays est avant tout celle d'un pays intérieur. Sony Labou Tansi, qui a passé toute sa vie au Congo, est l'écrivain de l'intérieur du pays, tandis que Tchicaya est l'œcoumène de ce ventre car il porte « l'insomnie du pays dans son ventre ${ }^{26} »$. Dans les deux cas, le ventre est le lieu de vie, celui à partir duquel ils écrivent.

C'est dans cette dynamique de vie, que le ventre maintient son unité, qu'il laisse épanouir sa puissance dans le langage fusionnel des corps qui peuvent alors remuer, danser, s'enivrer "pour la rumba / que je danse du torse / que tu danses des reins ». Dans «la ronde des mutins", les corps sont débordés par l'expressivité des flux multiples qui les traversent, et «la danse a, [comme le souligne Tchicaya U’Tamsi,] la meilleure langue / en laquelle faire de deux corps / les deux membres d'une phrase / dont il faut écrire au plus que parfait / le verbe aimer ${ }^{27}$ ».

Cette puissance du Ventre manifeste le surgissement du soi-collectif. Il devient le lieu de l'écriture de l'Histoire au moment où elle bégaye et dévoile l'effondrement de la bonne conscience : «j'épaule ma mémoire / et je tire dans le blanc des yeux ! / Soudain qu'ils soient ceux d'une femme / c'est moi qui meurs / sinon, ouvrir un ventre de femme / c'est ouvrir une tombe / ou la ronde pour une conga des mutins ${ }^{28}$ ", écrit encore TUT. 
21 C'est également le surgissement du tout jusqu'alors perdu dans ses parties, du Moyo disséminé clandestinement dans les ventres individuels qui en accueillent désormais les intensités, jusqu'à s'imaginer d'autres ventres pour une nouvelle fusion. L'auteur songe ainsi à ce :

[...] port

d'où nous partîmes

à la recherche d'un ventre commun

nous préservant de la fosse commune ${ }^{29}$.

Loin d'être une force englobante, niant les individualités, le ventre selon l'alternative conceptuelle qu'en offre Tchicaya U Tam'si, devient l'essence de l'être-ensemble. Il prend alors le caractère de la lutte - «le choix fut: / ou le combat ou l'opprobre ${ }^{30}$ ", constate l'écrivain -, accompagnée de la conscience de cette lutte : « Le ruissellement / obstinément lent du sang/partout où l'homme est passé /montre[, souligne le texte,] que toutes viscères dehors /le signal augural tue d'être $v^{31} »$... Car il est impossible de poser la légitimité du ventre indépendamment de l'objectivité historique, tout comme il n'est pas possible d'opposer purement et simplement la raison politique au ventre. La raison politique reste en effet impuissante, à partir du moment où elle ne s'accompagne pas d'affects ou ne se lie pas au ventre.

22 Aucune éthique n'est possible, dans ces conditions, qui ne se joue au cœur-même du ventre, dans l'opposition entre action et passion. Le ventre est la source de tous les affects, et spécialement de ceux qui incluent l'inclination violente vers un objet (Amour, aversion, rancune, haine...) :

L'amour désole

L'amour tue

L'amour s'en va.

Quand fut-il dit :

le ventre reste ${ }^{32}$ ?

se demande Tchicaya U'Tamsi.

«Quand fut-il dit: le ventre reste? ? : à cette question, il existe une première réponse qui clôture presque le recueil: «quand on ne sait plus / dans quelle nuit furent perdus / les corps et les biens d'une couronne / tout dans le dos et pour l'échine. Certes il reste le Ventre $»$.

23 Mais il en existe une seconde, que nous livre un des cinq personnages que Tchicaya $U$ Tam'si fait dialoguer dans « Les corps et les biens », l'avant-dernier chant de ce cahier : «On parle d'amour », déclare ainsi l'arpenteur en poursuivant : « on oublie le pain du peuple / dont la haine est le plus sûr levain / pour son pain quotidien ${ }^{33}$ ». D'où l'alternative : "Le pain ou La cendre », titre du recueil qui prolonge la problématique du ventre et dans lequel on trouve, à côté de l'arpenteur, le devin, le constructeur, le soldat et le poète.

Au poète qui assure : «N'a pas le Graal / ni chasubles d'or / qui n'a pas le ventre en creux $^{34}$ ", l'arpenteur rétorque: "Je ne quête pas le Graal / mais mon sang dans les rigoles de $\operatorname{Kin}^{35} »$.

Dans cette quête, le sang est le moyen d'accès direct à la géographie de la ville ${ }^{36}$, pour les lunaisons de «ces vierges honteuses de leur sang / qu'elles perdent sans jouir / à s'enfler le ventre ${ }^{37}$ ", souligne encore le poète qui, seul à recevoir «le sang des femmes / avec le secret des pires douleurs dans le ventre ${ }^{38}$ ", se tait, conscient de ce que, dans le récit de mort de cette chasse à l'homme, le contrepoint menstruel fonctionne comme un signal. 
Alors que le « ventre perd une lune ${ }^{39}$ après l'autre », insiste Tchicaya U Tam'si, « [...] un mur d'anciens ossements se lève, chante au pas cadencé un chant de partisans ${ }^{40} » . \mathrm{Ne}$ pouvant être tu, le ventre s'arrache progressivement aux forces extérieures pour dépendre de plus en plus fortement de la puissance de celui qui en est et reste le fondateur, Lumumba, devenu la figure de l'affirmation de soi afin d'éviter, souligne le texte, "la soumission / de tout ce qui est extrait du ventre ${ }^{41}$ !». Peu importe que l'affirmation de soi revienne à accepter sa propre mort, dans la confusion délirante et extatique de ces indépendances qui refoulent les difficultés d'être du ventre.

\section{Le prophète et la métamorphose du ventre} écrivains, devient donc indivisible.

Le dépositaire du pouvoir du nouveau ventre n'est plus l'oncle comme dans le lignage, mais celui qui peut transformer la quête d'indépendance postulée du ventre en une réalité dont il devient le garant, grâce à la recherche constante de l'unité. Et nul mieux que Lumumba n'en assume la volonté organisatrice dans l'histoire récente du pays.

La place de ce dernier, celle de porteur de ventre, ne tient pas du flux de sa parole, mais de ce que, souligne le texte, " dès les battements du tambour / on se le répète à Kin : / Le chant se drapa / de son visage ${ }^{42} »$. Lumumba est l'homme de l'économie du verbe : « Non, Avec le ventre, ramener tous les regards / à la souplesse unique de ma voix ${ }^{43}$ ", déclare-t-il. Parlant au nom du ventre et à partir de lui, il apparaît comme un ventriloque : «On m'assure qu'il fut ventriloque / alors les chacals se liguèrent / avec qui l'on sait ${ }^{44} \ldots$ », souligne le narrateur du Ventre - Le Pain ou La Cendre. La ventriloquie n'est pas synonyme d'engastrimythisme. Elle est ce qui qualifie le mieux le porteur de ventre : celui au nom et à partir duquel le ventre s'exprime.

Malheureusement, les faux prophètes, les chacals, parlent également (à partir) du ventre: "Avant qu'il ne fut fait à la terre / L'affront d'un galop de chacals / Hélas ventriloques ${ }^{45}$ !», déplore le narrateur -. D'où l'extrême confusion, le chaos funeste et la mort qui s'abat sur, souligne encore le texte : « le ventre / partout avec cette chaleur / pestilentielle des vieux charniers ${ }^{46} . .$. ».

C'est ce chaos qu'il est nécessaire de réfuter. La négation est le premier acte d'affirmation de soi. Le recueil Le Ventre s'ouvre ainsi par l'imprécation : " Non. / Je dis : non ${ }^{47}$ ", une posture individuelle, avant d'être celle, collective, du Ventre. Ceci, non en tant que négation de l'Autre, mais parce que, face à la sommation de répondre.

"Que le ventre réponde!», somme TUT : La reconnaissance de soi devient, ainsi, la manifestation existentielle de l'essence du ventre ${ }^{48}$, qui assure le passage de la pure négativité à la simple positivité qui en est le manifeste ${ }^{49}$.

Il dit :

Je dis à la femme

De ne vêtir son ventre que de grelots ...

Il dit :

Je dis : non! [souligne encore le texte.]

Ce n'est ainsi que dans l'incarnation du leader, que le ventre acquiert sa dynamique propre : «Je dis : non!». La spécificité de cette incarnation tient de ce que le ventre, organe anatomique, ne s'émancipe de sa morphogenèse que dans les contingences anthropologiques qui le fondent désormais comme organe social «dans les forges / où 
[note TUT] l'on battait le cuivre / pour les maillons / d'une chaîne d'hommes / unis du ventre et du cœur / dès l'œuf, dans tel giron ${ }^{50}$ ! »

La relation complexe qu'entretient la figure du leader avec le ventre est toujours une tension, car elle s'insère dans un rapport de forces où ce qui se maintient dans l'inachèvement de l'unité du ventre commande aussi son autonomisation. C'est à partir de ce rapport que le leader s'individualise et émerge du ventre comme chef et prophète ${ }^{51}$.

Là où l'on étouffait "sous un ventre / qui n'a pas su dire pardon / à l'ivraie plus guerrière / que l'abeille des champs ${ }^{52}$ ». Deux oppositions se dessinent : d'un côté, la représentation d'un ventre unificateur/uni, d'où est exclue toute scission et, de l'autre, la réalité d'un ventre travaillé de l'intérieur et de l'extérieur par l'insécurité et le souci névrotique de l'ordre.

Le ventre camoufle en effet des intériorités individuelles meurtries ${ }^{53}$. Tout comme le fondement de la liberté du Ventre (national) repose sur la mise à distance du ventre clanique, "ventre d'avant le Ventre », la réalisation totale du Ventre, "Ventre d'après le ventre " ou "ventre national ", n'est donc possible que par l'affirmation de soi comme inaliénable, dans un milieu où celui-ci se heurte à d'autres que lui.

Il en va ainsi du nombril, épicentre du ventre, organe mort qui se rappelle à nous comme résidu d'un temps d'avant le Ventre : "Tenant le nombril en laisse / le ventre ne sait plus / ce qu'il promet et à qui ", souligne encore le texte ${ }^{54}$. La figure du chef, dans le devenir-ventre du pays, se conçoit ainsi comme un être en déphasage. Le caractère "trouble-fête" du porteur de ventre ne tient qu'à ce qu'il proclame : "Je donne à mes frères leurs propres yeu $\mathrm{x}^{55} 》$, déclare ce dernier.

La lutte est le moment de ce déphasage. Afin que ventre se fasse, il faut en effet une procédure de différenciation dans l'unité de ceux qui le composent :

Rien n'est plus seul que le ventre -

Et le cœur !

Seul de cette solitude

Dont les saillies écorchent les plaies vives ;

Arrachent les dents de lait

Dès la première désillusion du cœur ${ }^{56}$ !,

souligne l'auteur qui, plus loin, note :

Que l'amour maternel

ne reprenne jamais du ventre

le corps qu'il donne du ventre...

Que le cordon ombilical rompu

le lien soit deux mains nouées

que l'on dénoue avant l'agonie...

A quoi bon rêver d'un ventre

qui soit aussi une tombe chaude ${ }^{57}$.

La critique du mythe des origines sera désormais constante dans l'écriture de TUT. Du moins telle qu'il la proclame dans son long poème La source:

Les sources dont je me méfie le plus sont celles qui ont été défigurées par l'histoire sanglante des hégémonies vicieuses. Ainsi les fontaines votives consacrées au culte d'ancêtres dont l'idiotie serait divinisable, par exemple.

Le placenta, première place d'armes

jeté

Le cordon ombilical, lien premier

Jeté.

C'est prescrit au rituel de la naissance. On a rompu aux deux extrêmes le cordon 
ombilical; on a noué le nombril. Une cicatrice témoigne de cette rupture sauvage. Si mon ventre la porte encore - comme un stigmate, une flétrissure - c'est une raison de plus de ne pas donner à ce rituel si barbare une valeur propitiatoire. Expulsé du ventre, jeté en pâture au monde, je chercherai toujours la lumière derrière laquelle me cacher, pour échapper aux vieux démons, aux vieux instincts. Grégaires ou autres ${ }^{58}$.

\section{Malentendus du ventre} sur la terre, ses limites, ses jalons, son histoire. Histoire Kongo, cela va de soi, mais pas seulement, insiste-t-il :

Nous avons toujours été les enfants du voyage, à telle enseigne qu'on a pensé que notre terre voyageait. Les Teke étaient devant, les Kongo (gens de paix) les suivaient, eux-mêmes étaient suivis par les Lwangu (aujourd'hui Vili). D'où la célèbre trilogie résumée par le proverbe connu de nous tous: Mute ngwoni, muko ngwoni, muvie ngwoni ngwoni, ce qui signifie, le ventre du Teke est le même ventre que le ventre du Kongo et celui du Vili (Ba Vila, ceux qui se sont perdus en sortant du Pays des Pyramides ou Pays des Rois ${ }^{59}$ ),

Cette divergence ne donnera pas lieu à une polémique, car elle repose sur un malentendu. Lorsqu'il rencontre Tchicaya U Tam'si, Sony Labou Tansi se demande « de quel droit cette grosse tête s'est mêlée de parler du ventre » et songe : "Sans doute parce que son ventre à lui est le lieu saint de la douleur ${ }^{60} »$. TUT prend alors ce dernier pour un « fieffé moralisateur ${ }^{61} » . .$. Loin de cette passe d'armes, la controverse porterait plutôt sur le tracé des frontières du Ventre.

Pour Tchicaya U Tam'si, s'inscrivant dans le dépassement du projet colonial, la finalité du Ventre est dans son universalité : dans le flux du fleuve qui se jette dans la mer. Cela amène Sony Labou Tansi à noter :

Tchicaya, c'était un vagabond, qui n'avait pas de pays. Tous les pays étaient son pays. Et il aimait de manière féroce le Congo. Enfin le Congo... Je ne sais pas lequel, parce qu'en plus il est allé au Congo belge ${ }^{62}$.

SLT en vient à égratigner la figure héroïque de Lumumba, lorsqu'il déclare à Bernard Magnier: «Lumumba n'avait aucune idée du Congo. L'idée que Lumumba avait du Congo lui avait été soufflée par les Belges, donc ça ne m'intéresse pas ${ }^{63} »$.

L'écrivain feint donc d'ignorer que la situation du porteur de ventre est instable, dictée par les conditions d'émergence historique de ce Ventre qui, non pleinement constitué, est déjà soumis à une instabilité chronique. Même glorieuse, l'histoire de la terre des gens du voyage, qu'il réhabilite, ne débarrasse, ainsi, aucunement cette terre, en mal de ventre, des ordres de mort imposée qu'U Tam'si avait perçue dans la tragédie de Lumumba ; et que lui-même finit par reconnaître dans Le quatrième côté du triangle, où il note : «Il était une fois / la terre / menteuse / douteuse / magouillée / bâclée ${ }^{64}$ » : une terre "boucanée, contradictoire", bien que congolisée "pour que la chose plaise à Dieu $^{65}{ }$; et qui s'interdit néanmoins à la vie.

Les critiques de Sony Labou Tansi se trouvent immédiatement compensées, comme chaque fois qu'il parle de Tchicaya U Tam'si, par une observation qui en absout les motivations : « Mais Tchicaya disait que chez nous, "le prince, c'est le fleuve". Pour lui, il n'y avait pas Mobutu, il n'y avait pas Sassou, il n'y avait personne. C'est le fleuve qui gouvernait et qui commandait ${ }^{66} "$, déclare-t-il dans Il Quarto lato del triangolo (Le Quatrième côté du triangle). 
'elle parle du flux des origines ou des destinées océanes des eaux du fleuve, la poétique du ventre, chez Sony Labou Tansi et chez Tchicaya U Tam'si, rend compte d'une dynamique où les forces qui la composent et la décomposent s'opposent de telle manière, qu'elles ne peuvent être pensées qu'à l'intérieur de cette opposition même... Comme si l'amour du pays et la vénération du ventre tenaient dans une passion, portée par l'opposition entre l'impétuosité des flots qui s'élancent vers la mer et le clapotis tempéré des eaux de ce fleuve taciturne :

Aimer l'Afrique, façon de parler [note Sony Labou Tansi] : Parler sans tout dire. La meilleure façon de se taire. L'eau a des grandes oreilles qui entendent tout. Des yeux qui semblent tout voir. Mais la gueule de l'eau est trop fine pour se perdre en paroles. Elle clapote de murmures savoureux ${ }^{67}$.

Tout comme "cette âme bannie [qui] clapote / tout le long du roulis ${ }^{68}$ " qu'évoque Tchicaya U Tam'si, dans le recueil plusieurs fois mentionné. C'est là, sur les berges d'un fleuve où s'échangent les souffles impétueux de ses courants et les balancements rythmés de ses clapotis, qu'a lieu leur rencontre.

Si le Tchicaya U Tam'si de « La Conga des mutins » est indissociable du projet du pays à bâtir avec la figure prométhéenne de Lumumba, celui du réel postcolonial affirme l'existence d'un noyau traumatique qui ne cesse de résister à la normalisation depuis que, souligne l'auteur: "Le ventre et la dépouille / désormais sans dialogue / perpétuent l'état de friche ${ }^{69} »$.

Cette friche est la conséquence de l'inachèvement du Ventre qui pousse Sony Labou Tansi à en dénoncer les méfaits, dans Sa Majesté le Ventre, sous la forme de la politique du ventre, décriée, des dirigeants africains. Cette tragi-comédie en deux tableaux met en scène le pantagruélisme du nouvel homme fort, qui ayant transformé la République en une monarchie à laquelle il ne peut accorder malheureusement de dauphin, se languit, souligne le texte, que « la mort d'un homme de [son] sang vienne seulement du ventre $^{70} »$. Toute la pièce tourne autour de la conjuration, dans un décor ubuesque, du sort de ce ventre :

Nous te vaincrons oh! Ventre ingrat

Nous te vaincrons nous te vaincrons

Chair inhumaine nous te vaincrons

Pauvre intestin et pauvre estomac

Pauvre caca et pauvre cœur [...].

Bien que ce soit le mal de ventre (Vumu) du pouvoir qui est ici visé, c'est sur le pays politique malade de son ventre (Moyo), que se concentre l'indignation des deux écrivains.

41 En 1977, après le coup d'état qui entraina la mort des Présidents Marien Ngouabi et Alphonse Massamba-Débat, ainsi que celui du Primat de l'Église catholique du Congo, Émile Biayenda, Sony s'écrie : «Un pays foutu / plus une terre pourrie / font naufrage / dans les poches / des compagnons d'armes / qui fusillent la vertu/ Ils ont fait l'inventaire / des ventres faméliques [...] $]^{11}$ ». Il ajoute à l'adresse d'Emile Biayenda : « Tu fis périr / ton ventre indigène / dans un vertige ténébreux / Ta chair / est entrée au silence / de la terre / kongolaisement promise ${ }^{72}$ ».

Comment ne pas lire, dans ces vers de Sony Labou Tansi, la même rage sombre qui parcourt "La mise à mort ", poème qui clôture Le Ventre de Tchicaya U Tam'si ? « Voilà ce pays s'en remet au crime / la boue et le sang emmêlent trois morts / un prêtre et deux séculiers de la géhenne ${ }^{73} »$. Un crime dont il fustige l'inutilité, avant d'implorer,

Continents manuscrits, 1 | 2014 
dans une ultime supplication : «Pour quoi te sort-il tous ces morts du ventre ${ }^{74}$ ?». Estce parce que " cette terre orgueilleuse / voulut / qu'on vînt l'irriguer / avec du sang ${ }^{75}$ ", comme se le demande Sony Labou Tansi ; ou parce que, selon les mots de Tchicaya U Tam'si : « La liberté nègre c'est finalement la mort par contumace ${ }^{76}$ »?

Parce que, comme il le souligne, "la liberté sans peine est une lubie ${ }^{77}$ ", le ventre à construire ne sera ni de l'ordre clanique, ni de l'héritage colonial, l'écrivain propose le dépassement d'une réalité associée autant au clanique qu'à l'héritage colonial, sous l'infléchissement du porteur de ventre, pourvu que celui-ci puisse s'autonomiser :

Car / telle est la vérité / l'Histoire se vend / mais ne s'achète pas-

L'avenir / jamais ne fut ramassé au coin de la rue...

On l'invente ${ }^{78}$.

Le ventre n'est donc pas seulement un thème littéraire, c'est la matrice de la singularité et de l'énergie d'écriture de Sony Labou Tansi et Tchicaya U Tam'si, qualifiés de prophètes. Le ventre travaille leur projet d'écriture, l'inscrit dans une histoire aussi bien collective qu'individuelle et y impose sa puissance anthropologique sur fonds d'horizon politique.

\section{Transduction, de la génétique à l'anthropologie}

43 Le ventre, par les glissements sémantiques et anthropologiques qui le travaillent, constitue un cas d'école pour l'étude des écrivains issus d'anciens pays colonisés. Ainsi, il s'agit d'examiner le passage d'une langue à l'autre, mais surtout d'une face de l'auteur à une autre. Dans ce cadre, il est nécessaire d'envisager une cohérence naturellement médiate, qui accompagne le transfert tout entier de son être écrivant là où, comme écrivain, il transporte une signification, l'accroît, la garantit et l'assume comme instance textuelle sémantique, qu'une approche anthropologique permet de mieux situer.

Le terme que l'on propose, pour expliciter ce phénomène est celui de " transduction »: un terme issu de la biologie, plus précisément de la génétique humaine ${ }^{79}$, où il désigne l'opération de transfert du matériel génétique d'une cellule à une autre par l'intermédiaire d'un vecteur viral ${ }^{80}$.

Son introduction en sciences humaines et sociales a bénéficié des travaux du philosophe Gilbert Simondon qui en a donné la définition suivante :

Nous entendons par transduction une opération physique, biologique, mentale, sociale, par laquelle une activité se propage de proche en proche à l'intérieur d'un domaine, en fondant cette propagation sur une structuration opérée de place en place : chaque région de structure constituée sert à la région suivante de principe de constitution, si bien qu'une modification s'étend ainsi progressivement en même temps que cette opération structurante ${ }^{81}$.

En génétique textuelle, l'activité " transductive " traduirait alors, dans le passage d'une langue à l'autre, la transfiguration d'un trope qui, en fonctionnant sur tous les registres qui lui permettent de conserver sa valeur originelle, se double, dans le nouveau répertoire, de sa puissance métaphorique. Il exige ainsi que nous abordions son sens médiat dans sa cohérence anthropologique, unique façon de prendre en compte cette transfiguration.

Dans ce transport, c'est l'écrivain qui, se situant à un niveau homodiégétique, migre tout entier avec les sèmes dont il a la charge. Il n'est pas hors du discours qu'il enrichit et dont il charge les mots des sèmes pluriels que la langue-hôte lui offre. L'efficacité de 
cette opération de transduction réside dans le renversement réflexif du sens car, dès que la langue-hôte règle le procès des termes employés en libérant toutes les significations qu'ils recèlent, la transduction cesse de se limiter à celles-ci. Certains sèmes que l'on croit les plus fondamentaux, subissent en effet, déjà, un "décentrage " dans la langue d'origine, tout en apparaissant dans le registre des significations de l'autre :

[Un d] écentrage accentué si l'on remonte le temps et l'on considère le rapport entre colonie et métropole. Décentrage doublé aussi, dans ce cas, d'une dimension politique et sociale de domination/soumission réelle et consciente pour l'auteur colonisé. Un rapport dont on peut ensuite se demander s'il persiste, a persisté ou non, de façon consciente ou inconsciente, chez les écrivains postcoloniaux ${ }^{82}$...

On ne saurait donc nier que ce qui, au premier chef, est l'enjeu de cette écriture, tient au rapport que l'écrivain africain francophone engage avec cette langue autre.

Cette spécificité révèle pourquoi certains termes ne semblent toujours fonctionner qu' «à distance ", c'est-à-dire grâce à une caution qui n'est, pourtant, jamais donnée pour soi, jamais présente autrement que dans son déplacement : «Et plus précisément, par une dialectique entre propriété et propriétés de la langue, entre son détenteur proclamé et son utilisateur ${ }^{83}$ ».

Il en résulte que c'est toujours à la périphérie que se situe l'écrivain africain francophone, parce que la langue française est, pour employer une expression de Jacques Lacan, le grand Autre de celui-ci qui ne peut l'aborder que par « une stratégie d'expression, mais aussi d'approche ou de conquête "du centre ${ }^{84 "}$ " .

Les deux notions, de « détenteur proclamé » et d'« utilisateur », sont, il va de soi, loin d'être symétriques, car détention et utilisation ne sont pas, elles-mêmes, comparables dans l'espace de l'appropriation de la langue de l'Autre. Dans sa dimension la plus fondamentale, le statut de la langue de l'Autre, en tant qu'institution symbolique, relèverait plutôt de l'utilisation que de la détention. L'utilisation est de l'ordre de la réalité, la détention est de l'ordre du fantasme,

[p]arce que le maitre ne possède pas en propre, naturellement, ce qu'il appelle pourtant sa langue ; parce que, quoi qu'il veuille ou fasse, il ne peut entretenir avec elle des rapports de propriété ou d'identité naturels, nationaux, congénitaux, ontologiques; parce qu'il ne peut accréditer et dire cette appropriation qu'au cours d'un procès non naturel de construction politico-phantasmatique, parce la langue n'est pas son bien naturel, parce que cela même il peut historiquement, à travers le viol d'une usurpation culturelle, c'est-à-dire d'essence coloniale, c'est-à-dire toujours d'essence coloniale, feindre de se l'approprier pour l'imposer comme la sienne ${ }^{85}$.

La détention est toujours réflexive, dans un chiasme signifiant où elle ne relève de la propriété que dans l'utilisation qui en est faite.

La détention, elle, n'équivaut pas nécessairement à l'utilisation, du fait qu'il peut toujours y avoir plusieurs façons d'utiliser un même support. C'est pourquoi, du fait du caractère réflexif inhérent à la propriété, l'écrivain africain peut s'approprier cette langue qui le distingue du « détenteur proclamé » par l'utilisation scripturaire qu'il en fait, pour s'établir dans son réel auctorial ${ }^{86}$.

L'écriture, graveuse de paroles, permet ainsi, en situation coloniale - et au-delà - de borner les déterminations d'une existence niée, assignée à ce signifiant qui répugne à prêter son identité. 
51 moyage avec des mots de sa langue en français, l'écrivain africain francophone n'a, ainsi, de cesse de signaler l'incomplétude de «sa » langue, face à celle de l'Autre ${ }^{87}$, dominante et unitaire. Mais, là où il sollicite du sens dans la langue de l'Autre, supposée disposer des significations qui lui manque, il découvre que ses requêtes restent sans solution, tournent autour d'un vide central qu'il ne peut combler que de sa propre langue.

Il se rassure alors avec les marques inscrites dans sa chair : des traces de souffrance, dont il fait un signifiant-maitre, qu'il produit comme contingence dans la langue de l'Autre, afin de mieux la remettre en cause. C'est sur ces gnosies, qui ne s'expliquent qu'à partir de ce point d'opacité, que surgit le verbe de l'écrivain, qui s'écrie : «J'écris (ou je crie) pour qu'il fasse homme en $\mathrm{moi}^{88}{ }^{\prime}$.

\section{NOTES}

1. Tchicaya U Tam'si, Le Ventre, Paris, Présence africaine, 1964 (l'édition de référence pour cet article sera sa réédition augmentée en 1978 de Le Pain et la cendre); Sony Labou Tansi, "Sa majesté le Ventre», avant-texte de Marcel Sony, Le Ventre, tapuscrit, concours théâtral inter-africain 1972, R/19446, DAEC, 29 mai 1972, 46 p. De ce dernier texte inédit, il existe une version manuscrite, non datée mais sans doute antérieure, publiée sous le titre original, «Sa Majesté le ventre », dans Sony Labou Tansi, L'autre monde. Écrits inédits, textes choisis par Nicolas Martin-Granel et Bruno Tilliette, Paris, Revue Noire, 1997.

2. Entretien avec E. Maunick, Demain l'Afrique, 1979.

3. « J'y ai mis mon ventre, mes entrailles, ma chair parce qu'il faut que les gens arrivent à respecter la vie à travers la chair... J'ai fait une dédicace à un ami. J'y ai mis : "Ce livre, c'est la place que le ventre demande sur une terre où la tête a trop pris". C'est à peu près ça que j'ai voulu exprimer dans mon livre ». Brouillon de "Lettre aux sagesfemmes d'une conscience: ou Messieurs les intellectuels, la science est-elle universelle? ", tapuscrit inédit, 12 août 1981.

4. « Enfin si les mots veulent / S'ils veulent / Prendre ventre / Et chausser le meilleur », Poème à G. Félix Tchicaya, Brazzaville, 1980.

5. Note manuscrite, non datée, Labou Tansi, 1997, p.77.

6. Propos recueillis par Caya Makhelé, Equateur, $n^{\circ} 1,1986$.

7. Sony Labou Tansi, "Je soussigné cardiaque», Le Journal de Chaillot (entretien), septembre 1985, p. 39.

8. Sony Labou Tansi, « Je soussigné cardiaque », Le Journal de Chaillot (entretien), ibid.

9. K.H. Leach, « La genèse comme mythe », Langages, 1971, Vol. 6, nº 22, p. 13-32 ; Pierre Boudrot, « Le héros fondateur », Hypothèses, 2001/1, p. 167-180.

10. Patrice Yengo, "Le rêve et la réalité. CEdipe lignager et mutations sorcières au Congo ", Rupture nouvelle série, Paris, Karthala, 2004, n 5, p.116.

Continents manuscrits, 1 | 2014 
11. Tchicaya U Tam'si, Le Ventre - Le Pain ou La Cendre, Paris, Présence africaine, 1978, p. 113.

12. Ibid, p. 114.

13. «Il n'est plus pensable qu'on puisse parler aux êtres humains sans en avoir le cœur tordu. Comment en ce $\mathrm{xx}^{\mathrm{e}}$ siècle ferait-on pour dire autrement qu'avec des mots tordus ». J'écris ce texte à l'intention de mon ami Pierre Debauche, un tordu de Belge qui, comme tous les créateurs, a la panse à gauche." (Avertissement, tapuscrit inédit, vers 1989).

14. parue aux éditions La Revue Noire en 2005.

15. Tchicaya U Tam'si, Le Ventre - Le Pain ou La Cendre, Paris, Présence africaine, 1978, p. 106.

16. Ibid., p. 96.

17. Ibid., p. 116.

18. Ibid., p. 46. «Il n'y a qu'à voir mes viscères... », souligne l'auteur.

19. Sony Labou Tansi, « Moi, Veuve de l'Empire », L'Avant-Scène, 1987, pp. 11, 15 et 32.

20. Tchicaya U Tam'si, Le Ventre - Le Pain ou La Cendre, op.cit., p. 58.

21. On retrouve cette vision chez Georg Groddeck, " Du ventre humain et de son âme ", Nouvelle Revue de psychanalyse, $\mathrm{n}^{\circ} 3,1971, \mathrm{p} .211-247$.

22. Tchicaya U Tam'si, Le Ventre - Le Pain ou La Cendre, op.cit., p. 45.

23. Ibid., p. 97.

24. Archives SLT, Note prise sur un agenda, (N. Martin-Granel), Brazzaville, vers 1978.

25. Boniface Mongo-Mboussa Boniface, « Écrire le Congo à partir de l'ailleurs : Tchicaya U Tam'Si », Africultures, 03/2002.

26. Tchicaya U Tam'si, Le Ventre - Le Pain ou La Cendre op.cit., p. 144.

27. Ibid., p. 23.

28. Tchicaya U Tam'si, Le Ventre - Le Pain ou La Cendre, ibid., p. 35.

29. Ibid., p. 38.

30. Ibid., p. 59.

31. Ibid., p. 69.

32. Ibid., p. 18.

33. Tchicaya U Tam'si, Le Ventre - Le Pain ou La Cendre, ibid., p. 130.

34. Ibid., p. 131.

35. Ibid., p. 132.

36. Ibid., p. 39. « Kitona ou Kamina/Congolais !/Le sang le sang le sang », note l'auteur.

37. Ibid., p. 58.

38. Ibid., p. 77.

39. Dans les langues kongo, voir ou perdre une lune pour une femme signifie avoir ses menstrues.

40. Tchicaya U Tam'si, ibid., p. 70.

41. Ibid., p. 48.

42. Ibid., p. 25. 
43. Ibid., p. 24.

44. Tchicaya U Tam'si, ibid.

45. Ibid., p. 20.

46. Ibid.

47. Ibid., p. 13.

48. Ibid., p. 26 : « Le ventre répond du ventre », souligne le texte.

49. Ibid.

50. Ibid., p. 14.

51. Cf. Tchicaya U Tam'si, ibid., p. 40.

52. Ibid., p. 18.

53. Ibid., p. 69 : «C'est ainsi que le corps / pour lequel onctions et massages /furent prescrits - du dedans les termites - du dehors les limaces perlèrent sans combler les crevasses d'ordures. ", souligne le texte.

54. Ibid., p. 15.

55. Ibid., p. 26.

56. Ibid., p. 22.

57. Ibid., p. 67.

58. Tchicaya U Tam'si, « La source », Revue noire, $\mathrm{n}^{\circ} 5$, p. 54.

59. "Les sources Kongo de mon imagination », 24 avril 1993, in Jean-Michel Devesa, Sony Labou Tansi : écrivain de la honte et des rives magiques du Kongo, Paris, L'Harmattan, 1996, p. 359.

60. Sony Labou Tansi : «Tchicaya U Tam'si : le père de notre rêve », in Notre Librairie $\mathrm{n}^{\circ}$ 92-93, mars-mai 1988.

61. Tchicaya U Tam'si, « Portrait d'un écrivain par un autre », Equateur, nº 1, p. 10.

62. Entretien avec Bernard Magnier, 26 octobre 1993, in Sony Labou Tansi. Paroles inédites, Éditions Théâtrales, 2005, p. 55.

63. Ibid.

64. Sony Labou tansi, Il Quarto lato del triangolo (Le Quatrième côté du triangle), édition bilingue (français/italien), La Rosa, coll. « Tracce », 1997, p. 156.

65. Il quarto lato del triangolo, ibid., p. 140.

66. Ibid.

67. Ibid., Jeune Afrique Economie, $\mathrm{n}^{\circ} 141$.

68. TUT, op. cit., p. 61

69. Ibid., p. 68.

70. L'autre monde. Écrits inédits, Éditions Revue Noire, Paris, 1997, p. 81.

71. Il quarto lato del triangolo, op. cit., p. 6.

72. S. Labou Tansi, « Pour Emile Biayenda » in Il quarto lato del triangolo (Le quatrième côté du triangle), p. 64.

73. TUT, op. cit., p. 162.

74. Ibid., p. 156.

75. Il quarto lato del triangolo, ibid., p. 70. 
76. TUT, ibid., p. 166.

77. Ibid., p. 165.

78. Ibid., p. 38.

79. Norton D. Zinder et Joshua Lederberg furent les premiers à en faire usage dans un article de référence: "Genetic exchange in salmonella", Journal of Bacteriology, 1952 November, 64(5), p. 679-699.

80. R. Cunin, Génétique bactérienne, Vigot, Paris, 1993.

81. Gilbert Simondon, L'individuation à la lumière des notions de forme et d'information, Grenoble, éd. J. Millon, coll. « Krisis », 2005, p. 34. Noté désormais ILFI. Ibid., p. 32. Cette définition est issue de l'étude de la genèse d'un cristal qui constitue le paradigme de l'individuation pour Simondon. Il lui arrive de l'amender, notamment pour penser l'individuation vitale et psychique. Comme l'a noté Jean-Hugues Barthélémy, cette universalité partielle du paradigme cristallin est problématique, notamment pour lui accorder une validité analogique.

82. Jean-Pierre Orban, «Interférences et création. La dynamique auteur-éditeur » dans le processus de création chez Sony Labou Tansi à partir de la comparaison entre Machin la Hernie et L'Etat honteux ", Genesis n' 33, 2011, p. 30.

83. Jean-Pierre Orban, ibid., p. 30.

84. Ibid.

85. Jacques Derrida, Le monolinguisme de l'autre, Paris, Galilée, 1996, p. 45.

86. Maurice Couturier, "Pour une lecture intersubjective du roman", La figure de l'auteur, Paris, Le Seuil, « Poétique », p. 242.

87. Voir, à ce propos, le dernier essai d'Alain Mabanckou, Le sanglot de l'homme noir, Paris, Fayard, 2012, p. 190. Toute autre est, ceci dit, la position de SLT.

88. Ifé Orisha, «Sony Labou Tansi face à douze mots ", Équateur, n ${ }^{\circ} 1$, Paris, octobrenovembre 1986, p. 29.

\section{RÉSUMÉS}

L'auteur francophone n'est pas un simple traducteur; son positionnement d'écrivain dans une langue qui n'est pas la sienne brouille les contours de cette fonction. On voudrait ici rendre compte de cette dynamique singulière, à partir du champ sémantique du ventre, central dans l'œuvre de Tchicaya U Tam'si et de Sony Labou Tansi, auquel ces deux auteurs ont consacré deux de leurs ouvrages au titre homonyme, le Ventre (1964) et Sa majesté le ventre (1972).

A French-speaking author is not a simple translator of his culture into French: his positioning of the writer in a language which is not his mother tongue, blurs the outline of translator's craft. The article presents this particular dynamics using the semantic field of the belly, which is central in the literary production of Tchicaya U Tam'si and Sony Labou Tansi. Both authors devoted to this theme their homonymous writings: Le Ventre (The Belly, 1978) and Sa Majesté le ventre (His Majesty, the Belly, 1972). 
INDEX

Mots-clés : analyse génétique, anthropologie, Congo-Brazzaville, Sony Labou Tansi, Tchicaya U Tam'si, ventre, traducteur, multilinguisme

Index géographique : Congo-Brazzaville

\section{AUTEUR}

PATRICE YENGO

Université de Brazzaville (Rép. Congo) 